Arthroskopie 2009 $\cdot 22: 6-7$

DOI 10.1007/s00142-008-0496-5

Online publiziert: 16. Januar 2009

(c) Springer Medizin Verlag 2009

\author{
R. Seil · D. Pape \\ Centre de l'Appareil Locomoteur, de Médecine du Sport et de \\ Prévention, Clinique d'Eich, Centre Hospitalier de Luxembourg
}

\title{
Das kindliche Kniegelenk
}

\section{Entwicklung}

„Das kindliche Kniegelenk“ umfasst die Lebenszeitspanne von der Geburt bis zum Verschluss der Wachstumsfugen, unabhängig vom numerischen Lebensalter. Hierbei handelt es sich jedoch nicht um das Gelenk ,kleiner Erwachsener“, die ein bestimmtes Lebensalter noch nicht erreicht haben, sondern um ein Gelenk mit spezifischen anatomischen und physiologischen Eigenschaften. Es sind v. a. die kniegelenknahen Wachstumsfugen, die dafür verantwortlich sind, dass man beim kindlichen Kniegelenk auch die zeitliche Dimension und hier bevorzugt das skelettale Alter bei den therapeutischen Strategien berücksichtigen muss. Etwa 2/3 des Längenwachstums der unteren Extremität stammen aus diesen Fugen. Sie haben eine hohe Wachstumsgeschwindigkeit, die während des pubertären Wachstumsschubes im Vergleich zum Wachstum des Rumpfes nur minimal zunimmt. Danach nimmt sie rapide ab und das Längenwachstum des Kniegelenks kommt vor dem Wachstum des Rumpfes zum Stillstand. Parallel hierzu kommt es zu einer Ausreifung der Kapsel- und Bandstrukturen. Vor der Pubertät ist die physiologische Gelenklaxität viel höher als beim Erwachsenen. Sie gleicht sich gegen Ende des kniegelenknahen Wachstums der von den meisten Arthroskopeuren bekannten Laxität des Erwachsenen an. Verletzungen, Entwicklungsstörungen und Fehlstellungen während des Längenwachstums unterscheiden sich dementsprechend nach Art, Prognose und Therapie deutlich von denen des Erwachsenenalters. Neben den oben genannten Faktoren liegt dies auch an der größeren Heilungs- und Korrekturpotenz des wachsenden Skeletts.

\section{Derzeitige Lage}

Ein weiterer Faktor, der es rechtfertigt, eine ganze Ausgabe dieser Zeitschrift dem kindlichen Kniegelenk zu widmen, liegt im folgenden gesellschaftlichen Paradoxon:

Einerseits sind über 7 Mio. Kinder und Jugendliche in einem der Verbände des Deutschen Sportbundes regelmäßig körperlich aktiv. Durch die zunehmende Leistungsstimulierung im frühen Alter (siehe Horizont der olympischen Jugendspiele) und der steigenden Zahl an Risikosportarten sind diese Kinder besonders verletzungsgefährdet. Auf der anderen Seite sind heute bereits 30\% Kinder übergewichtig und fettleibig und bei vielen sind zunehmend schlechtere motorische Fähigkeiten festzustellen. Die Konsequenzen hieraus sehen wir in unserer täglichen Praxis mit einer zunehmenden Anzahl an schweren Knieverletzungen im jugendlichen Alter. Sport- und Freizeitverletzungen stellen derzeit mit mehr als $30 \%$ aller Verletzungen die häufigste Verletzungsursache im Kindes- und Jugendalter dar. Das Kniegelenk ist dabei das zweithäufigste verletzte Gelenk.

\section{Beiträge in diesem Heft}

Im Beitrag von Theisen et al. wird die Epidemiologie der kindlichen Kniegelenkverletzungen dargestellt und auf zahlreiche Risikofaktoren (Alter, Vorverletzung, Sportart und -kontext, Trainingsumfang, Koordination sowie psychologischer Zustand) für Sportverletzungen hingewiesen. Einige dieser Faktoren sind beeinflussbar und sollten in die primäre und sekundäre Verletzungsprävention einbezogen werden.
Osti et al. erläutern die prognostische Bedeutung und das diagnostische und therapeutische Vorgehen bei kniegelenknahen Frakturen des distalen Femurs und der proximalen Tibia im Kindes- und Jugendalter, die sich aus extraartikulären metaphysären Frakturen, Frakturen mit Beteiligung der Wachstumsfugen und knöchernen, rein epiphysären Bandausrissen zusammensetzen.

In den letzten Jahren haben sich die arthroskopischen Therapieverfahren rasant weiterentwickelt (vgl. „Arthroskopie“, Heft 11, 1998) und sind nicht mehr vergleichbar mit der bloßen diagnostischen Arthroskopie und Spülung eines posttraumatischen Hämarthros, wie sie in der frühen 8oer Jahren praktiziert wurde.

Im Beitrag von Lorbach et al. werden die schwierige Diagnostik kindlicher Meniskusläsionen erläutert und die unterschiedlichen Ursachen eines instabilen Meniskus erklärt. Die Naht des kindlichen Meniskus erscheint ebenso Erfolg versprechend wie die des Erwachsenen.

Wilmes et al. sowie Kopf et al. widmen sich den immer häufiger festgestellten Rupturen des vorderen Kreuzbandes im Kindes- und Jugendalter. Diagnostische Eigenschaften, natürlicher Verlauf der Verletzung, Indikationsstellung der Operation, Rekonstruktionstechniken und postoperativer Verlauf werden eingehend dargestellt. Aufgrund der schlechten klinischen Ergebnisse nach konservativer Therapie besteht heute Konsens, dass auch bei Patienten mit offenen Wachstumsfugen eine VKB-Rekonstruktion zur Stabilisierung des Kniegelenks erfolgen sollte.

Jung et al. gehen auf neuere Erkenntnisse der nicht seltenen Osteochondrosis dissecans ein. Auch im Bereich der schwierigen patellofemoralen Problematik kommt es zunehmend zu neueren Er- 
kenntnissen und Therapieformen. Sie werden eingehend in den Arbeiten von Schoettle et al. und Schneider et al. beschrieben.

Dieses Heft soll dem Leser einen Überblick über die Epidemiologie und Therapie kindlicher Kniegelenkverletzungen geben. Wir hoffen, dass die hervorragend ausgearbeiteten Beiträge dieses Heftes auch dem erfahrenen Orthopäden und Traumatologen in der täglichen Praxis von Nutzen sind.

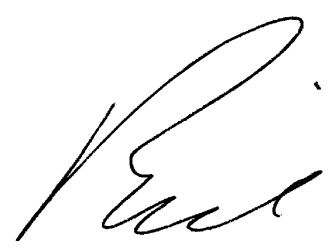

Prof. Dr. Romain Seil<smiles>C1=CCCC1</smiles>

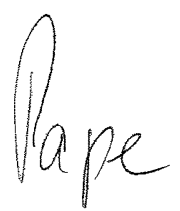

PD Dr. Dietrich Pape

\section{Korrespondenzadresse}

\section{Prof. Dr. R. Seil}

Centre de l'Appareil Locomoteur, de Médecine du Sport et de Prévention, Clinique d'Eich, Centre Hospitalier de Luxembourg 78, rue d'Eich, L-1460 Luxembourg rseil@yahoo.com

\section{Schmerztherapie „von der Stange" kann riskant sein}

Bekanntermaßen kann dieselbe Menge eines Schmerzmittels bei einem $\mathrm{Pa}$ tienten hervorragend anschlagen, bei einem anderen aber lebensbedrohliche Nebenwirkungen hervorrufen. Eine Studie der Universität Bonn zeigt nun, welche Rolle genetische Varianten bei der Schmerzbekämpfung spielen.

Weil das menschliche Schmerzempfinden sehr subjektiv ausfällt, ist es für Ärzte in der Regel schwer, den tatsächlichen Schmerzmittelbedarf zu ermitteln. Mit Hilfe einer Schmerzmittelpumpe, die nach größeren Operationen häufig eingesetzt wird, kann dieser Bedarf genauer quantifiziert werden. Die Patienten können ihr Schmerzmittel per Knopfdruck selber abrufen. Der subjektive Schmerzmittelbedarf kann bei einem bestimmten Eingriff um den Faktor 20 bis 50 variieren.

In der in Bonn durchgeführten Studie konnten die Teilnehmer über eine solche Schmerzmittelpumpe entscheiden, wie viel Tramadol sie über eine Infusion bekamen. Die Forscher stellten fest, dass die Menge des zugeführten Schmerzmittels unter anderem von der genetischen Ausstattung des jeweiligen Patienten abhing. Ein hoher Verbrauch an Tramadol in den 48 Stunden nach einer Operation hing signifikant mit einer bestimmten genetischen Variante des CYP2DA zusammen, die,Poor Metabolizer' genannt wird.“ „Poor Metabolizer" produzieren nur wenig Enzym des Typs CYP2D6, das für die Verstoffwechselung zahlreicher Medikamente verantwortlich ist. Das Enzym wandelt u.a. die Schmerzmittel Tramadol und Codein in ihre wirksamen Formen O-Demethyltramadol und Morphin um. Die schmerzlindernde Wirkung für diese beiden Medikamente ist dadurch herabgesetzt. Im Gegensatz dazu bewirkt die CYP2D6-Variante „Ultra Rapid Metabolizer" genau das Gegenteil bewirkt. Besonders viele Enzyme werden synthetisiert. Bei Menschen mit dieser Variante setzt die Schmerzmittelwirkung von Tramadol und Codein sehr schnell und ausgeprägt ein. Allerdings birgt sie auch ein überdurchschnittlich hohes Risiko für Nebenwirkungen.

Für diese Genvarianten gibt es regionale Unterschiede in der Verteilung. So taucht die CYP2D6-Genvariante „Ultra Rapid Metabolizer" bei Skandinaviern nur mit einer Wahrscheinlichkeit von etwa zwei Prozent auf, während fast ein Drittel aller Äthiopier diese Genversion hat.

Auf der Grundlage dieser Ergebnisse befürworten die Bonn Forscher eine verstärkte Individualisierung der Schmerztherapie. Vor allem bei Patienten mit chronischen Schmerzen und bei solchen, die gleichzeitig mehrere Medikamente einnehmen, sollten genetische Tests durchgeführt werden, die derzeit für die tägliche Routine aber noch zu teuer sind.

Originalpublikation:

Stamer UM, Stüber F, Muders T et al. (2008) Respiratory depression with tramadol in a patient with renal impairment and CYP2D6 gene duplication. Anesth Analg. 107(3):926-9

Quelle: Universität Bonn, www.uni-bonn.de 